\title{
Ten-year Evolution Utilizing Computer-Assisted Reconstruction for Giant Ameloblastoma
}

\author{
P. Niclas Broer, MD ${ }^{1} \quad$ Neil Tanna, MD, MBA ${ }^{1} \quad$ Peter B. Franco, DMD ${ }^{1} \quad$ Vishal D. Thanik, MD ${ }^{1}$ \\ Steven M. Levine, MD ${ }^{1}$ Evan S. Garfein, MD ${ }^{1}$ Pierre B. Saadeh, MD ${ }^{1}$ Daniel J. Ceradini, MD ${ }^{1}$ \\ David L. Hirsch, MD, DDS ${ }^{1}$ Jamie P. Levine, MD ${ }^{1}$
}

${ }^{1}$ Institute of Reconstructive Plastic Surgery, New York University, New

York, New York, USA

Address for correspondence and reprint requests Jamie P. Levine, MD, 530 First Avenue, Suite 8Y, New York, NY 10016, USA

| Reconstr Microsurg 2013;29:173-180. (e-mail: Jamie.levine@nyumc.org).

\begin{abstract}
Background The authors describe our current practice of computer-aided virtual planned and pre-executed surgeries using microvascular free tissue transfer with immediate placement of implants and dental prosthetics.

Methods All patients with ameloblastomas treated at New York University (NYU) Medical Center during a 10-year period from September 2001 to December 2011 were identified. Of the 38 (36 mandible/2 maxilla) patients that were treated in this time period, 20 were identified with advanced disease (giant ameloblastoma) requiring aggressive resection. Reconstruction of the resultant defects utilized microvascular free tissue transfer with an osseocutaneous fibular flap in all 20 of these patients.

Results Of the patients reconstructed with free vascularized tissue transfer, 35\% (7) 20) developed complications. There were two complete flap failures with consequent contralateral fibula flap placement. Sixteen patients to date have undergone placement of endosteal implants for complete dental rehabilitation, nine of which received immediate placement of the implants at the time of the free flap reconstruction. The three most recent patients received immediate placement of dental implants at the

Keywords

- ameloblastoma

- mandible reconstruction

- free fibula time of microvascular free tissue transfer as well as concurrent placement of dental prosthesis.

Conclusions To our knowledge, this patient cohort represents the largest series of comprehensive computer aided free-flap reconstruction with dental restoration for giant type ameloblastoma.
\end{abstract}

Odontogenic tumors are a complex and diverse group of lesions affecting the maxillomandibular skeleton. Classification of these lesions is based upon their embryonic tissue origin-namely epithelial, mesenchymal, or mixed. Ameloblastomas account for $1 \%$ of all oral tumors and $10 \%$ of all tumors from odontogenic origin., ${ }^{1,2}$

Surgical treatment of ameloblastomas depends on the size and location of the tumor, but for large or recurrent tumors, resection of the involved bone is the treatment of choice. For small defects, local resection, curettage, and bone grafting may be performed. For so-called "giant" or "extreme" ameloblastomas, a microvascular free flap with bone is often required. Although there is no strict definition, these are lesions that have uniformly become exophytic, disrupting normal anatomy, and are greater than $6 \mathrm{~cm}$ in size. Such tumors will always require composite resection to obtain clear margins and stabilization with vascularized bone and rigid fixation plating. Mandibular reconstruction utilizing a received

May 6, 2012

accepted after revision

September 7, 2012

published online

December 28, 2012 
free fibula flap is used preferentially by the authors. Twenty patients are presented in this report that required resection of giant type ameloblastomas of either the mandible or maxilla and underwent reconstruction with a composite free fibula transfer.

The goal in all of these patients is to not only resect the tumor but to provide an orthognathically ideal reconstruction. In this report, the authors present their evolving experience of computer-aided, computer-aided design and computer-aided manufacturing (CAD/CAM) virtually planned precise bony and dental restoration for these tumors. Initially, the hallmark of the preoperative approach had been meticulous preoperative planning of all parts of the procedure utilizing three-dimensional (3D) stereolithographic models. However, over the past 10 years this evolution has taken us to our current practice of virtual surgical simulation utilizing reconstructed 3D images and creation of staged models and cutting guides, which enable precise osteotomies and plating, providing for an ideal functional outcome.

\section{Methods}

All patients with ameloblastoma treated at the Institute of Reconstructive Plastic Surgery at New York University were included in this study. The 10-year study period extended from November 2001 to December 2011. A multidisciplinary team evaluated all patients.

Thirty-eight patients with ameloblastoma of the maxilla or mandible that met the inclusion criteria were identified. The mean age of these 38 patients was 32 ; the majority were male ( $n=26)$. Of these 38 patients, 20 were found to have giant type ameloblastomas. With institutional review board (IRB) approval, the charts and records of all these patients were reviewed. Excluded from the study $(n=2)$ were patients with incomplete records (examinations, photographs, or documentation) and inadequate follow up.

Patient demographics were noted for each patient. Additionally, the records were reviewed for type and length of resection, resultant bony and soft tissue defects, tumor pathology, type and timing of reconstruction, specifics of the reconstruction, stage of implant reconstruction and complications.

\section{Results}

Twenty patients presented with giant or extreme ameloblastoma of the upper and lower jaw. Eighteen patients underwent resection for mandibular tumors and two for maxillary pathology. The size of the resected lesions ranged from 5 to $15 \mathrm{~cm}$. Eight patients underwent segmental mandibulectomy and 10 underwent hemimandibulectomy. Two patients required total condylar resection and reconstruction due to the tumor size and location. A total of 22 free fibular flaps were placed. For all flaps a skin island was included in the harvest to utilize either repairing mucosal defects or for soft tissue augmentation.

Sixteen patients to date have received endosseous dental implants, nine of which received immediate placement of the implants at the time of the free flap reconstruction. The three most recent patients received immediate placement of dental implants as well as immediate placement of a dental prosthesis. All patients who received endosteal implants resulted in near normal function including range of motion, mastication, and phonation when compared with their preresection level of function. Immediate placement of the implants at the time of resection has become our standard of care for treatment of benign tumors unless technically problematic. ${ }^{3}$

Seven of the 38 patients experienced some type of complication. There were two flap failures secondary to arterial thrombosis that required removal and replacement with the fibula from the contralateral lower extremity. There was also one partial flap loss, which occurred in the distal portion of a double-barreled segment. This area was subsequently resected and not consequential toward full bone healing. The patient eventually proceeded to implant placement and full restoration. There were three donor site complications, of which temporary traction injury to the common peroneal nerve was the most serious but eventually had full recovery. Two patients experienced skin graft breakdown, which required local dressing care but healed without further intervention. Also there was one neck hematoma requiring reexploration.

\section{Reconstruction Protocol}

The size of the tumor in all patients was so large that the normal anatomic contour of the mandible or maxilla was completely lost. For the initial 11 cases, model surgery was performed on the stereolithographic skeleton. The resection was executed on this model and the plate bent precisely into the desired position, taking into account the appropriate height for the fibula and the desired mandibular shape. This plate was subsequently sterilized and utilized in the operating room (OR) for the patient's reconstruction. Because of the exophytic nature of the tumor, it was often impossible to place the final plate prior to the resection, requiring strategies for jaw stabilization during the surgery. This usually included a series of plates bent around the tumor analogous to an external fixator (-Fig. 1).

Most recently, we have advanced our techniques with Medical Modeling Inc. (Golden, Colorado, USA) by performing the resection and reconstruction virtually and creating the plates and models entirely based off the computed tomography (CT) scans and computer models of dental occlusion. This precisely planned virtual surgery enabled fabrication of plates and intermediary splints, bone cutting guides, and virtual and subsequent life placement of endosseous dental implants and dental prosthetics. ${ }^{4}$ As a result, the sites of the planned osteotomies and reconstruction are now accounted for in the virtual surgery; the stereolithographic model is created with the free bone graft in place, which can then be used to precisely contour the plates.

\section{Complete Computer-Aided Surgical Modeling}

Computer-aided mandibular reconstruction involves a planning phase, a modeling phase, and the surgical phase. 


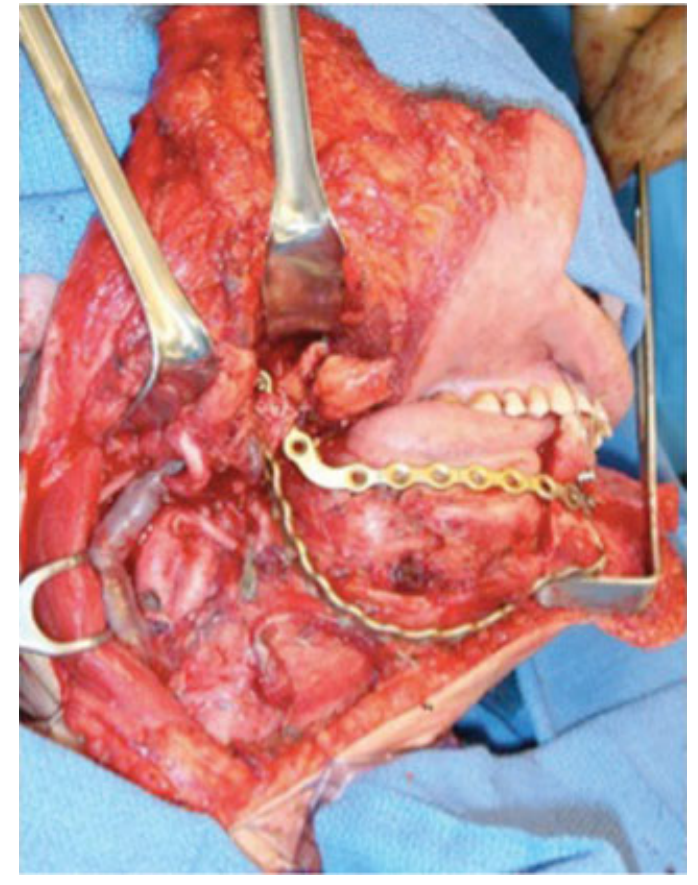

Fig. 1 To provide jaw stabilization during surgery, a series of plates are bent around the exophytic tumor analogous to an external fixator.

\section{Planning Phase}

Planning begins with a high-resolution CT scan of the patient's craniofacial skeleton and scans of the lower extremities to obtain information about the vascular and bony anatomy. The images are then forwarded to the modeling company (Medical Modeling, Inc.). The scans are converted into 3D, virtual reconstructions. A web meeting is then held with engineers from the modeling company and the surgical team. The key parameters of the planning phase are the margins of bony resection and location of fibula placement in relation to the remaining mandible or maxilla. These parameters are determined by the surgeon and marked on the $3 \mathrm{D}$ reconstruction image (-Fig. $\mathbf{2}$ ).

After cutting paths are chosen at the margins of the diseased bone, the segment is virtually removed and the planning of the reconstruction begins. The 3D fibular image is superimposed on the mandibular defect in its desired orientation. Using this image, fibular osteotomies are designed to fit the idealized reconstruction, thus recreating the shape of the resected portion of the native, nondiseased mandible or maxilla. The shape of the plate and the number and lengths of fibular segments can be modified online to optimize the shape of the reconstruction; maintain well-vascularized segments of fibula; provide an appropriate bone-plate relationship for positioning of implants; seamless bony approximation; and, most importantly, maintain a perfect occlusal arrangement. Although the fibula provides excellent support and bone width, the height of the fibula is less than that of the mandible. ${ }^{5}$ Consequently, when planning the placement of the fibula, we take into account the desire for orthodontic restoration with endosteal implants by positioning the bone more on the central to upper portion of the mandible to facilitate placement of the implants.

\section{Modeling Phase}

Next, the modeling phase begins, which involves stereolithographic manufacturing of the planned components. A model of the native craniofacial skeleton is created to assist intraoperatively. Cutting guides are produced that fit onto the mandible and allow the angles of resection to exactly match those created during the planning phase. A reconstruction plate template is designed that facilitates precise bending of the titanium plate preoperatively and can be made to match the plate design of the desired plating company.

\section{Surgical Phase}

The harvest of the osseocutaneous flap is performed in typical fashion. As noted above, there is often a gingival defect with resection of giant ameloblastomas, and harvest of a skin paddle provides security of a tension-free intraoral closure. In general, when an intraoral skin paddle is required, the contralateral fibula is chosen as the donor site. The cutaneous perforators are identified using a handheld Doppler preoperatively. The skin paddle size is estimated based on the need for intraoral and extraoral soft tissue. Harvesting of the flexor hallucis longus or a portion of the soleus muscle, if needed, obtains additional bulk. The length of the pedicle is increased by dissecting the peroneal artery and veins away from the fibula to the point where the osteotomies are to be performed.

\section{Technique for Performing the Osteotomies}

Initially, we planned the osteotomies using a technique of cutting a disposable ruler intraoperatively to act as a template for the fibula osteotomies ( $\mathbf{- F i g . ~ 3 A}$ ). The wedges that are cut were used to guide the wedges to be taken from the fibula and the remaining, straight segments guided the length needed for each fibula segment (-Fig. 3B). However, as outlined, this approach has now been modified and modernized significantly.

The challenge of judging the appropriate fibula lengths and intersection angles has become obviated by the use of the fibula cutting guides, which, as stated above, are fabricated from the virtual fibula and provide cutting slots that guide the osteotomies and exactly replicate the cuts for both the endand closing-wedge osteotomies as planned (-Fig. 4). After completion of these osteotomies, the fibula is secured to the reconstruction plate. The need for trimming of the neomandible should be minimal because the cutting guides reproduce the exact angles for a flush bone-to-bone fit (-Fig. 5A, B).

Taking these techniques a step further, we now, prior to creation of the fibular osteotomies, use the nSequence Company (Reno, Nevada, USA) endosseous implant guides to place dental implants in the fibula. The planning phase is similar to before but has become more sophisticated ( - Fig. 6A, B). The models are created with the implant and dentures in place (-Fig. 7A, B). This makes it possible to have the dental prosthesis immediately loaded onto the dental implants while the flap is still attached to the vascular pedicle ( $\mathbf{- F i g . ~ 8 A - C ) . ~}$

Regarding the facial portion of the procedure, incisions are planned and the resection is begun simultaneously to the flap harvest. Access to the mandible or maxilla is based on location and tumor size and, after this is obtained, placing the dentate patient into maxillo-mandibular fixation (MMF) 

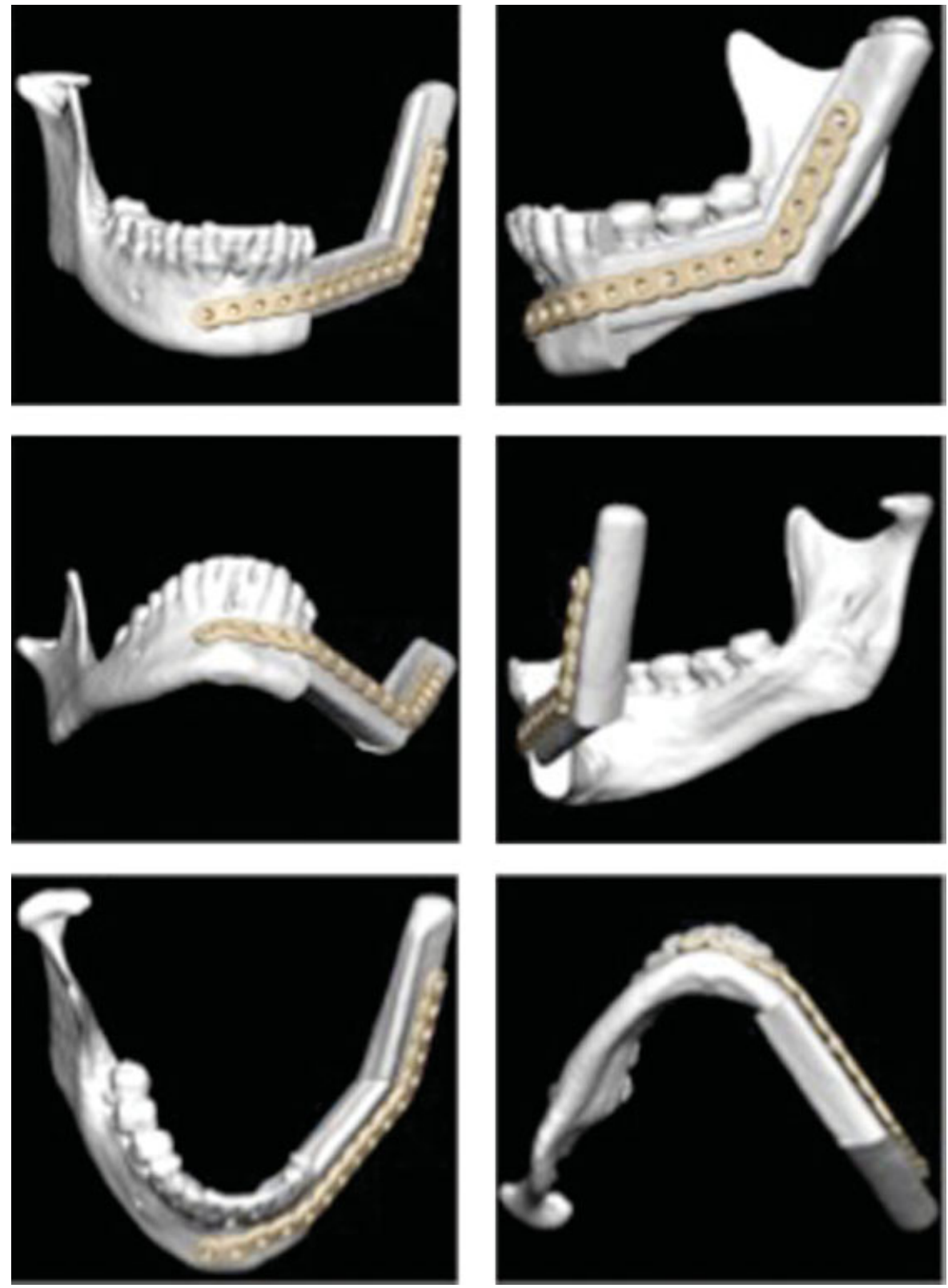

Fig. 2 Images are reconstructed in three-dimensional (3D) to provide the margins of bony resection and location of fibula placement in relation to the remaining mandible or maxilla.

or the Luhr technique (attaching the proximal mandible to the maxilla) are performed. ${ }^{6}$ Once the diseased bone segment is exposed and the proximal and distal segments are stabilized, the mandibular cutting guides are then secured to the mandible (-Fig. 9). These guides enable an exact duplication of the angles of osteotomy that were planned on the computer. A sagittal saw is then used through these jigs to create the osteotomies. Once the mandible is resected, the definitive reconstruction plate is placed on the mandible in the predetermined position.

The flap is then divided from the lower extremity, brought to the mandibular defect, and secured to the previously placed reconstruction plate. The dentures and a splint and intermaxillary fixation (IMF) are used to further maintain the patient in occlusion, maximizing both efficiency and precision (-Fig. 10).
The anastomoses in the neck are performed in standard fashion and can be performed on the side of the mandibular defect or, in the case of central or bilateral defects, the side where better quality vessels are found.

\section{Discussion}

Ameloblastomas are generally benign, but locally invasive, tumors of odontogenic origin.

Classification of these lesions is based upon morphology. Ameloblastomas are placed into five subtypes:

1. Solid, the most common type, is characterized radiographically by a multilocular appearance and histopathologically by connective tissue invasion. ${ }^{7,8}$

2. Cystic luminal ameloblastoma is characterized by the existence of a single cystic cavity lined by ameloblastic 
A
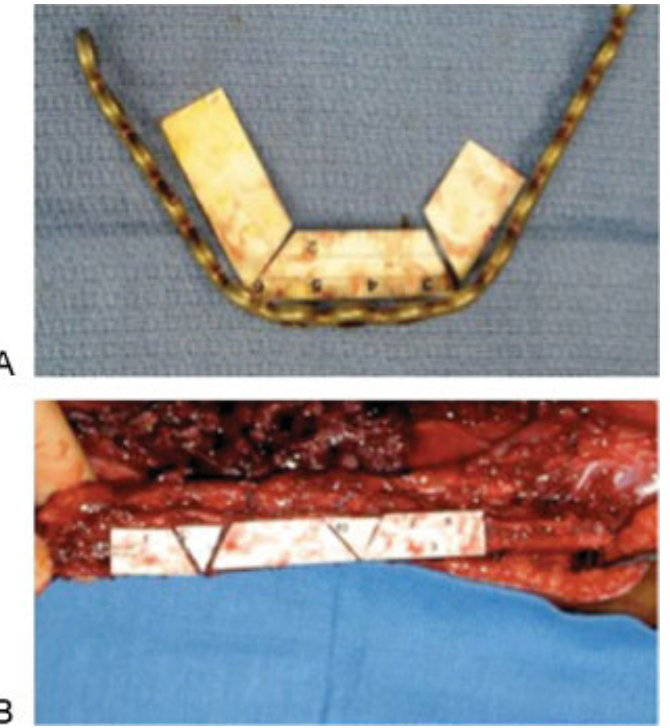

Fig. 3 (A) Mandibular steotomies are conventionally planned with an intraoperative ruler to act as a template for the fibula osteotomies.

(B) The wedges from the cut ruler are transposed to the donor site to guide the osteotomies to be done on the fibula.

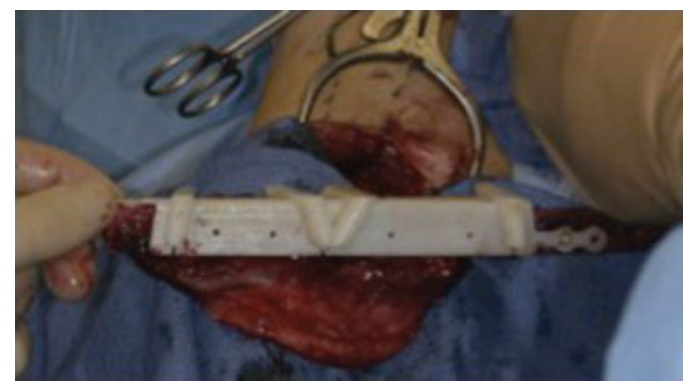

Fig. 4 Fibula cutting guides provide cutting slots that guide the osteotomies exactly as preoperatively planned.
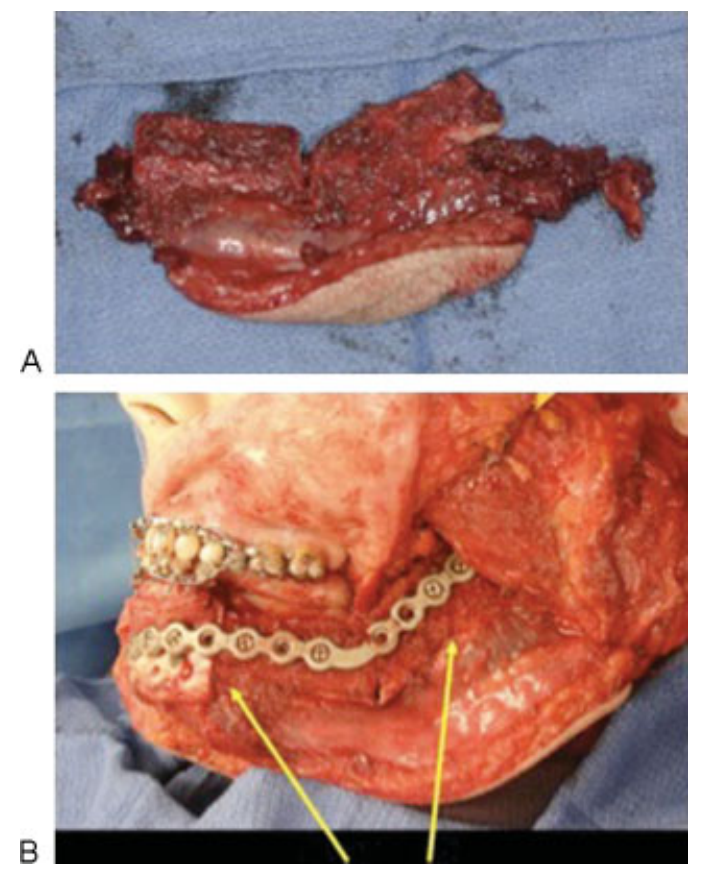

Fig. 5 (A) The cutting guides allow for efficient harvest of an osteocutaneous donor flap. (B) The flap is ready to be secured to the reconstruction plate with a flush bone to bone fit with the native mandible.

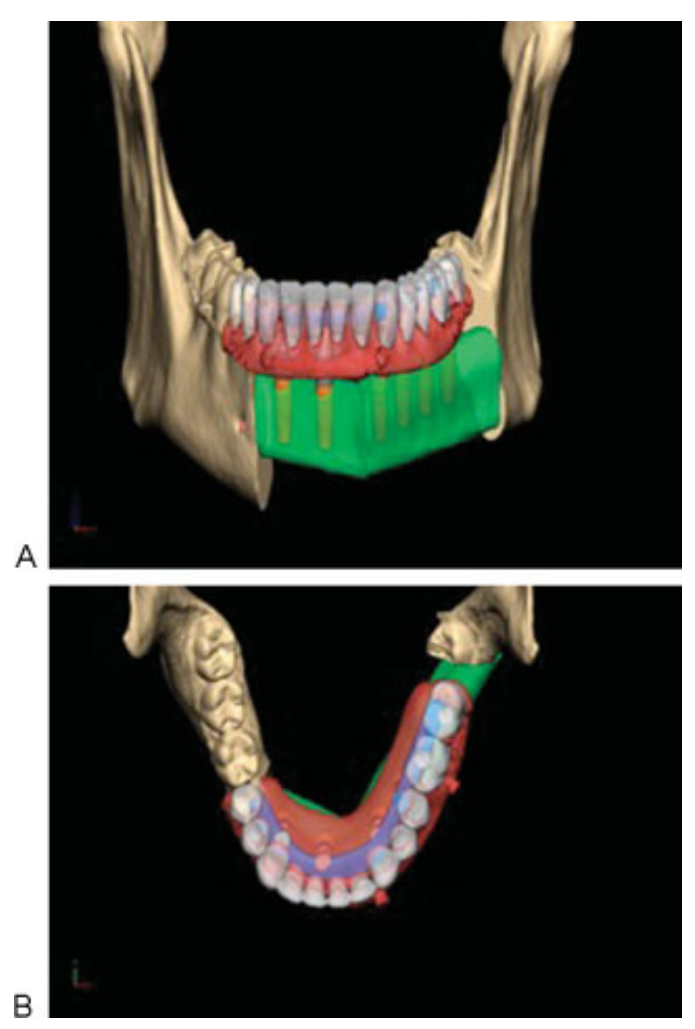

Fig. 6 (A, B) In select patients, during preoperative modeling, dental implants can be planned to be placed at the time of fibula flap harvest.

epithelium and growth of neoplastic tissue into the cystic lumen. Adolescent patients more often present with this type, which is often associated with an impacted third molar. Treatment of these lesions is generally conservative. $^{8,9}$

3. Cystic mural ameloblastoma is characterized by the growth of ameloblastic tissue from the connective tissue of the cystic lining or ameloblastomatous transformation of the entirety of the epithelial lining. This type is thought to be more aggressive than the cystic luminal subtype.,

4. Extraosseous or peripheral ameloblastoma affects the oral soft tissues, especially the gingiva. ${ }^{8}$ This subtype is typically found in the retromolar mandibular region in patients in the fifth and sixth decades of life. The tumor is superficial to the bone and treated with local excision. ${ }^{10}$

5. Desmoplastic ameloblastoma is characterized by compressed islands and thin cords of ameloblastic or basaloid epithelial cells within collagenized stroma. Unique to the desmoplastic variant, this tumor has poorly demarcated borders radiographically. This subtype requires complete resection. ${ }^{9}$

Rarely ameloblastomas may exhibit malignant behavior, as in the cases of ameloblastic carcinoma ${ }^{7}$ and malignant ameloblastoma. ${ }^{11}$

Approximately $80 \%$ of ameloblastomas occur in the mandible, most often in the molar/ascending ramus regions. The remaining $20 \%$ of lesions occur in the posterior maxillary region. 8,12 Typically, ameloblastomas are asymptomatic. Smaller lesions are often only detected upon routine radiographic examination. As the tumor enlarges, patients report a 
A

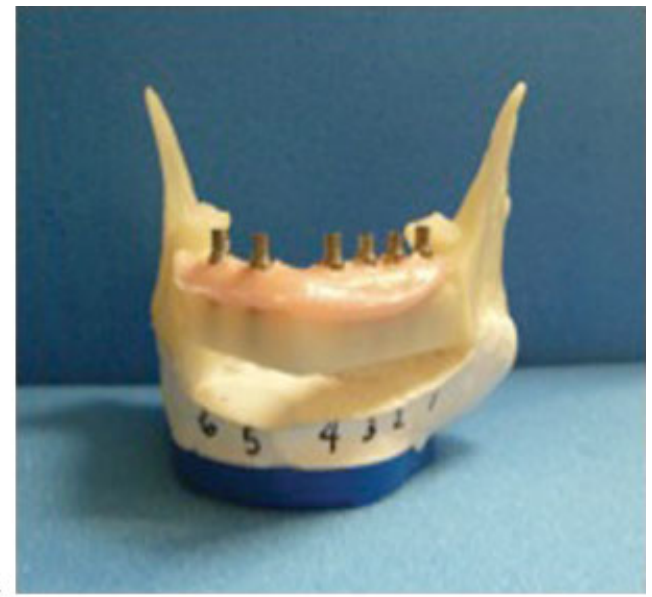

B

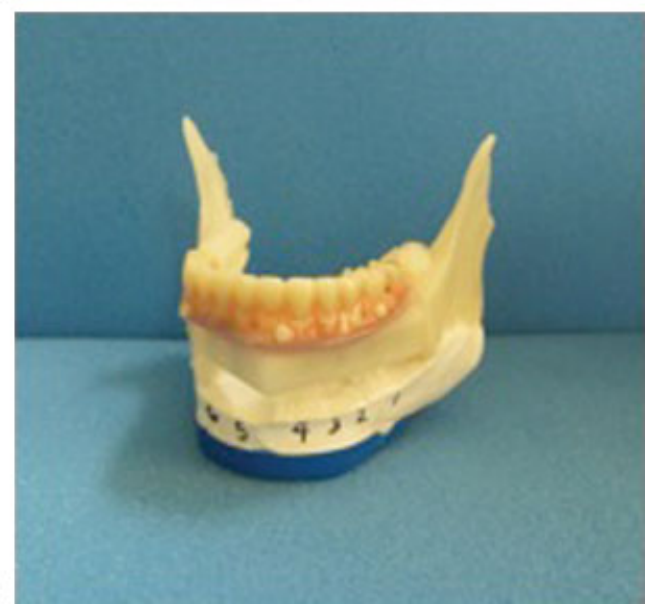

Fig. 7 (A, B) Models are created with the implant and dentures in place.

slow-growing, painless swelling, typically of the posterior jaws, with no history of trauma or carious teeth in the region. Paresthesias are rare. ${ }^{13}$ The most typical radiographic presentation of this neoplasm is that of a multilocular radiolucency associated with an unerupted tooth. Features of buccal/ lingual cortical expansion-mobility of teeth, delayed eruption of teeth, displaced teeth as well as the resorption of roots of adjacent teeth-are common. ${ }^{12,14}$

Treatment options range from curettage to local excision to en bloc resection of the affected bone. Resection is usually reserved for large solid lesions or cystic lesions demonstrating aggressive clinical behavior. Treatment options of smaller, cystic ameloblastomas include curettage, chemical destruction (i.e., cryotherapy/Carnoy's solution), and marsupialization/decompression. ${ }^{15,16}$

Giant type ameloblastoma-related lesions are often exophytic and can distort the normal anatomy significantly. Because of the cosmetic and functional importance of the face, the reconstructive demands are considerable, often necessitating vascularized bone flaps. ${ }^{17}$ Selection of the ideal flap for mandibular reconstruction depends on the specific requirements of the patient.

The three most commonly used options are the fibula, scapula, and iliac crest. ${ }^{18}$
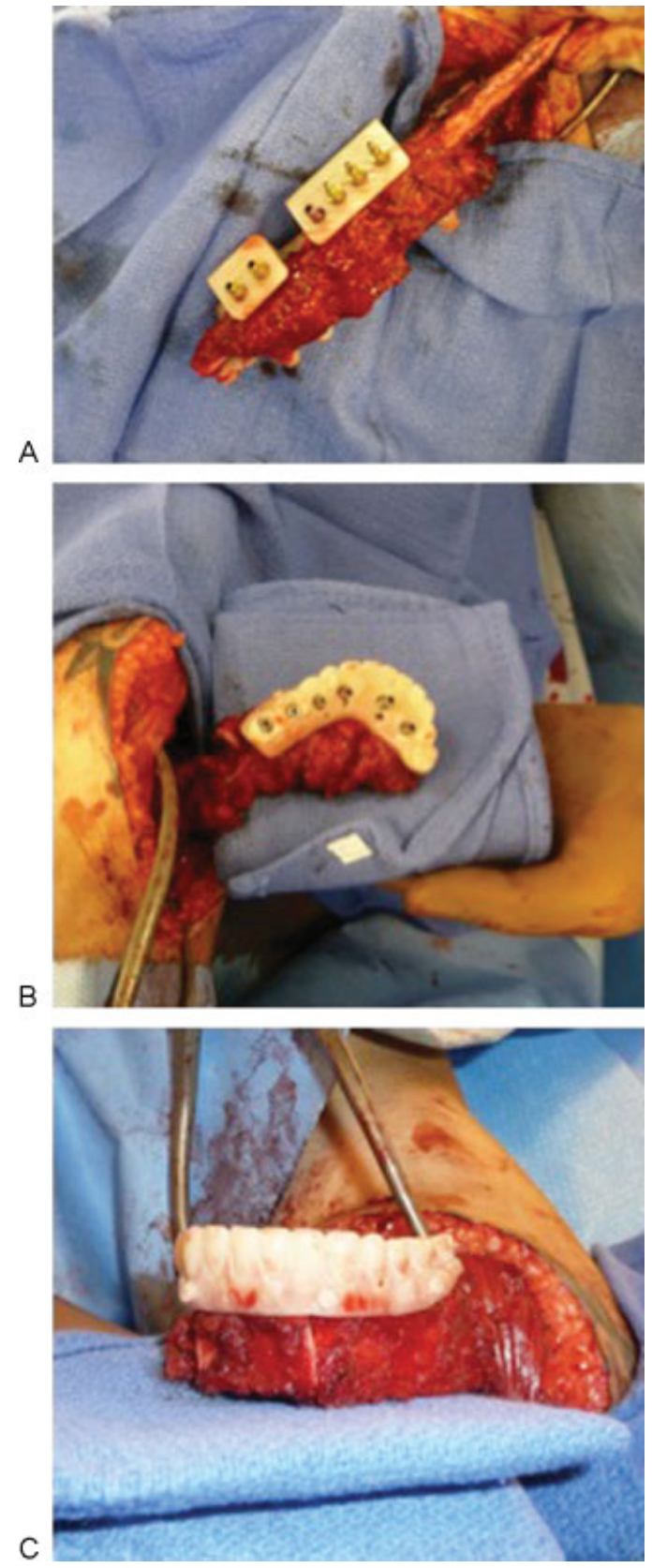

Fig. 8 (A, B, C) For patients who will have the dental prosthesis immediately loaded onto the dental implants, this can all be done while the flap is still attached to the vascular pedicle.

Since Hidalgo's initial report (1989), ${ }^{19}$ the osseous or osseocutaneous free fibula flap has emerged as the gold standard for mandibular reconstruction. This flap provides many advantages over previously described methods. ${ }^{20-23}$ It has a large, reliable vascular pedicle. The dual endosteal and segmental periosteal circulation allows the reconstructive surgeon to perform multiple osteotomies to shape the neomandible without devascularizing the flap. Length and width of the fibula permit immediate or delayed placement of osseointegrated implants. Furthermore, it can be harvested by a second team simultaneous to the mandible resection.

Even though ameloblastomas are benign, the gingiva that remains after resection is often unreliable and usually 


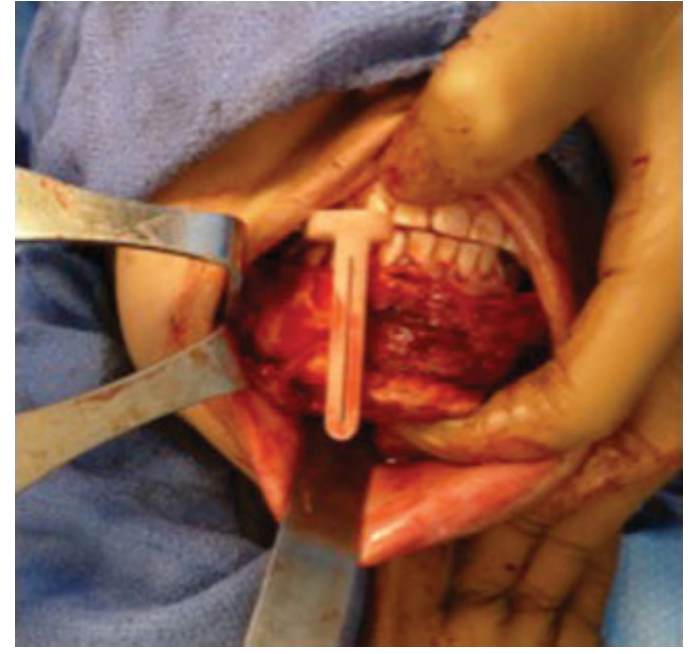

Fig. 9 The mandibular cutting guides are secured to the mandible to assist in tumor extirpation.

requires replacement with an intraoral skin island. The osseocutaneous fibula flap provides for a large, thin, pliable skin island, which can be harvested based on septocutaneous perforators. This paddle also helps to protect the bone, plate, and neck from intraoral secretions and allows for easier flap monitoring postoperatively. The two complete flap failures out of the 22 flaps occurred early in our series, but since we have used a combination of skin paddles and implantable arterial and venous Doppler monitoring, we have not encountered another flap loss. It can, if bulky, be removed postoperatively to provide for easier implant placement and creation of a buccal sulcus. One disadvantage when including such a skin island is the frequent necessity of skin grafting the donor site. ${ }^{24}$

Over the course of the past 10 years, we have significantly modified our approach when addressing these tumors. Similar to others, in our center, operations that require free flap reconstruction are performed by two teams: the extirpative and the reconstructive. The length of the procedure can thus be minimized by having the two teams perform as much of the operation as possible simultaneously. With the introduction of 3D laser stereolithography, exact models of the craniofacial skeleton can be produced rapidly and with rela-

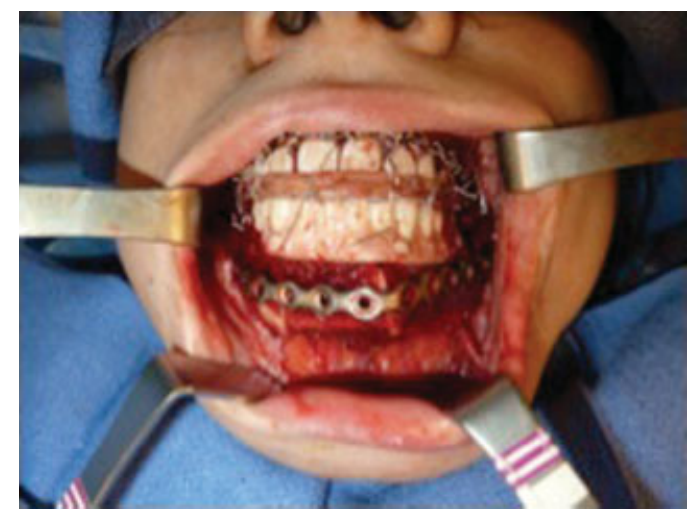

Fig. 10 Oromandibular reconstruction with dentition is performed in a single stage. tive ease. We utilize these models whenever bony reconstruction is indicated, which has made preoperative planning and plate conformation considerably easier and faster. This results in shortened operative time by eliminating intraoperative plate bending and maximizes both functional and aesthetic results. Initially, we also used these models to perform model surgery and provide an exact template for designing the fibular osteotomies. Now, however, the entire operationincluding the fibula osteotomies and inset-are performed virtually, and appropriate cutting guides are designed accordingly as outlined above.

Although we have been transitioning over the past 4 years to a complete CAD/CAM pathway of treatment for all of our head and neck reconstructive patients, we only evolved these techniques based off of the precision in surgical planning that was offered by using the stereolithographic models. The incorporation of these models has changed, for the better, the surgical planning, operative time, and most importantly the functional outcomes of patients. ${ }^{25,26}$ The same techniques utilized for the planning of the osteotomies are now also used to plan the exact location of the endosteal implants. To date, 15 patients have gone on to complete dental restoration. Eight of these patients had endosteal implants placed intraoperatively at the time of reconstruction, ultimately leading to a reduced number of interventions required.

\section{Conclusion}

This report describes our experience in treating 20 patients with giant type ameloblastomas. We describe the evolution of our techniques from using preoperatively obtained 3D stereolithographic models of the cranial skeleton to perform model surgery to our current concept of an complete CAD/CAMguided approach. At our institution, resection and microvascularized free flap reconstruction with immediate placement of endosseous dental implants and dentures has become the standard for ameloblastomas larger than $6 \mathrm{~cm}$.

\section{References}

1 Macanovic M, Gangidi S, Porter G, Brown S, Courtney D, Porter J. Incidental bony pathology when reporting trauma orthopantomograms. Clin Radiol 2010;65(10):842-849

2 Olaitan AA, Arole G, Adekeye EO. Recurrent ameloblastoma of the jaws. A follow-up study. Int J Oral Maxillofac Surg 1998;27:456460

3 Wu Y-Q, Huang W, Zhang Z-Y, Zhang ZY, Zhang CP, Sun J. Clinical outcome of dental implants placed in fibula-free flaps for orofacial reconstruction. Chin Med J (Engl) 2008;121:1861-1865

4 Thankappan K, Trivedi NP, Subash P, et al. Three-dimensional computed tomography-based contouring of a free fibula bone graft for mandibular reconstruction. J Oral Maxillofac Surg 2008;66:2185-2192

5 Bähr W, Stoll P, Wächter R. Use of the "double barrel" free vascularized fibula in mandibular reconstruction. J Oral Maxillofac Surg 1998;56:38-44

6 Marchetti C, Bianchi A, Mazzoni S, Cipriani R, Campobassi A. Oromandibular reconstruction using a fibula osteocutaneous free flap: four different "preplating" techniques. Plast Reconstr Surg 2006;118:643-651 
7 Ramesh RS, Manjunath S, Ustad TH, Pais S, Shivakumar K. Unicystic ameloblastoma of the mandible-an unusual case report and review of literature. Head Neck Oncol 2010;2:1

8 Rapidis AD, Andressakis DD, Stavrianos SD, et al. Ameloblastomas of the jaws: clinico-pathological review of 11 patients. Eur J Surg Oncol 2004;30:998-1002

9 Black CC, Addante RR, Mohila CA. Intraosseous ameloblastoma. Oral Surg Oral Med Oral Pathol Oral Radiol Endod 2010;110: 585-592

10 Vanoven BJ, Parker NP, Petruzzelli GJ. Peripheral ameloblastoma of the maxilla: a case report and literature review. Am J Otolaryngol 2008;29:357-360

11 Dao TV, Bastidas JA, Kelsch R, Kraut RA. Malignant ameloblastoma: a case report of a recent onset of neck swelling in a patient with a previously treated ameloblastoma. J Oral Maxillofac Surg 2009;67:2685-2689

12 Reichart PA, Philipsen HP, Sonner S. Ameloblastoma: biological profile of 3677 cases. Eur J Cancer B Oral Oncol 1995;31B:86-99

13 Sham E, Leong J, Maher R, Schenberg M, Leung M, Mansour AK Mandibular ameloblastoma: clinical experience and literature review. ANZ J Surg 2009;79:739-744

14 Adeline VL, Dimba EA, Wakoli KA, et al. Clinicopathologic features of ameloblastoma in Kenya: a 10-year audit. J Craniofac Surg 2008;19:1589-1593

15 Mendenhall WM, Werning JW, Fernandes R, Malyapa RS, Mendenhall NP. Ameloblastoma. Am J Clin Oncol 2007;30:645-648

16 Ghandhi D, Ayoub AF, Pogrel MA, MacDonald G, Brocklebank LM, Moos KF. Ameloblastoma: a surgeon's dilemma. J Oral Maxillofac Surg 2006;64:1010-1014
17 Pogrel MA, Podlesh S, Anthony JP, Alexander J. A comparison of vascularized and nonvascularized bone grafts for reconstruction of mandibular continuity defects. J Oral Maxillofac Surg 1997;55:1200-1206

18 Takushima A, Harii K, Asato H, Nakatsuka T, Kimata Y. Mandibular reconstruction using microvascular free flaps: a statistical analysis of 178 cases. Plast Reconstr Surg 2001;108:1555-1563

19 Hidalgo DA. Fibula free flap: a new method of mandible reconstruction. Plast Reconstr Surg 1989;84:71-79

20 Lutz BS, Wei FC. Microsurgical workhorse flaps in head and neck reconstruction. Clin Plast Surg 2005;32:421-430, vii

21 Kim EK, Evangelista M, Evans GR. Use of free tissue transfers in head and neck reconstruction. J Craniofac Surg 2008;19:1577-1582

22 Moro A, Cannas R, Boniello R, Gasparini G, Pelo S. Techniques on modeling the vascularized free fibula flap in mandibular reconstruction. J Craniofac Surg 2009;20:1571-1573

23 Cordeiro PG, Disa JJ, Hidalgo DA, Hu QY. Reconstruction of the mandible with osseous free flaps: a 10-year experience with 150 consecutive patients. Plast Reconstr Surg 1999;104:1314-1320

24 Peled M, El-Naaj IA, Lipin Y, Ardekian L. The use of free fibular flap for functional mandibular reconstruction. J Oral Maxillofac Surg 2005;63:220-224

25 Ndukwe KC, Adebiyi EK, Ugboko VI, et al. Ameloblastic carcinoma: a multicenter Nigerian study. J Oral Maxillofac Surg 2010;68:2111-2114

26 Darshani Gunawardhana KS, Jayasooriya PR, Rambukewela IK, Tilakaratne WM. A clinico-pathological comparison between mandibular and maxillary ameloblastomas in Sri Lanka. J Oral Pathol Med 2010;39:236-241 